\title{
Analysis of the strain induced martensitic transformation in austenitic steel subjected to dynamic perforation
}

\author{
J.A. Rodríguez-Martínez ${ }^{1}$, A. Rusinek ${ }^{2}$, R. Pesci ${ }^{3}$, and R. Zaera ${ }^{1}$ \\ 1 Department of Continuum Mechanics and Structural Analysis, University Carlos III of Madrid, Avda. de la \\ Universidad, 30, 28911 Leganés, Madrid, Spain \\ 2 Laboratory of Mechanics, Biomechanics, Polymers and Structures (LaBPS), National Engineering School of \\ Metz (ENIM), Route d'Ars Laquenexy, CS65820, 57078 Metz Cedex 3, France \\ 3 ENSAM-Arts et Métiers ParisTech, Laboratoire d'Études des Microstructures et de Mécanique des Matériaux \\ LEM3, UMR CNRS 7239, 4 rue Augustin Fresnel, 57078 Metz Cedex 3, France
}

\begin{abstract}
An experimental and numerical analysis on the martensitic transformation in AISI 304 steel sheets subjected to perforation by conical and hemispherical projectiles is reported. Two target thicknesses are considered, 0.5 and $1.0 \mathrm{~mm}$, and impact velocities range from 35 to $200 \mathrm{~m} / \mathrm{s}$. The perforation mechanisms are identified and the effect of the projectile nose-shape on the ability of the target for energy absorption is evaluated. Martensite has been detected in all the impacted samples and the role played by the projectile nose-shape on the transformation is highlighted. A 3D model implemented in ABAQUS/Explicit allowed to simulate the perforation tests. The material is defined through a constitutive description developed by the authors to describe the strain induced martensitic transformation taking place in metastable austenitic steels at high strain rates. The numerical results are compared with the experimental evidence and satisfactory matching is obtained. The numerical model succeeds in describing the perforation mechanisms associated to each projectile-target configuration analysed.
\end{abstract}

\section{Introduction}

Steel sheets are cost-efficient and easily affordable materials, with valuable properties for the industry. In some industrial applications, they are prone to suffer dynamic indentation or perforation: high-speed blanking and hole flanging in manufacturing processes, or impact by strikers in vehicles, vessels, marine hulls and shields. Thus, the deformation and damage initiation/development in the target are relevant phenomena which should be considered when assessing the performance of steel sheets against impact loading. Several papers have been published on the perforation of thin plates by blunt, conical or hemispherical projectiles. Corbet et al. [1] did a thorough review on penetration and perforation of plates at sub-ordnance velocities, covering literature from 70's, 80's and early 90 's. The research in this field was likewise very active during the last two decades as suggested by the number of published papers.

Hemispherical perforation in sheets produces a circumferential neck followed by circumferential cracking (discing). As the impact velocity further increases a hinged cap is separated from the sheet and remains attached. Higher impact velocities produce a clean separation and ejection of the cap, and short radial cracks. Conical strikers cause failure through radial cracking tearing out into a number of petals, their number increasing with impact velocity and projectile nose angle. Energy absorption is then driven by a combination of processes: bending and stretching of the plate (structural response), fracture and petal curling (local response). Most of the published works deal with aluminium, mild steel, copper or brass. Only a few of them $[2,3]$ consider metastable austenitic steel alloys exhibiting Strain Induced Martensitic Transformation (SIMT). These grades have the ability to transform from the initial face-centered cubic austenite phase to body-centered cubic martensite during plastic deformation. The hardness of martensite is usually higher than that of austenite, increasing yield stress and strain hardening of the two-phase steel. Additionally, the martensitic transformation causes the Transformation Induced Plasticity (TRIP) effect, and this enhances the work hardenability of the steel, delays the onset of necking, and thus improves formability.

This work presents an experimental and numerical analysis on the martensitic transformation in AISI 304 steel sheets subjected to perforation by conical and hemispherical projectiles. Perforation tests are carried out using a pneumatic gas gun within the range of impact velocities 35 to $200 \mathrm{~m} / \mathrm{s}$. The experimental arrangement enables the determination of impact velocity, residual velocity and failure mode of the steel sheets. It has been detected that the SIMT occurs during perforation for all the tests performed. A 3D model implemented in ABAQUS/Explicit allowed to simulate the perforation tests. The material is defined through a constitutive model developed by the authors to describe the strain induced martensitic transformation taking place in metastable austenitic steels at high strain rates. The finite element results are correlated with the experimental evidence and satisfactory agreement is found for all the projectile-target configurations examined. The roles played by impact velocity, target thickness and projectile nose shape on the martensitic transformation are properly captured.

\section{Specimens and experimental set-up}

The material studied in this work is the AISI 304 austenitic stainless steel. The AISI 304 is considered as the reference metastable austenitic stainless steel for studying the SIMT 


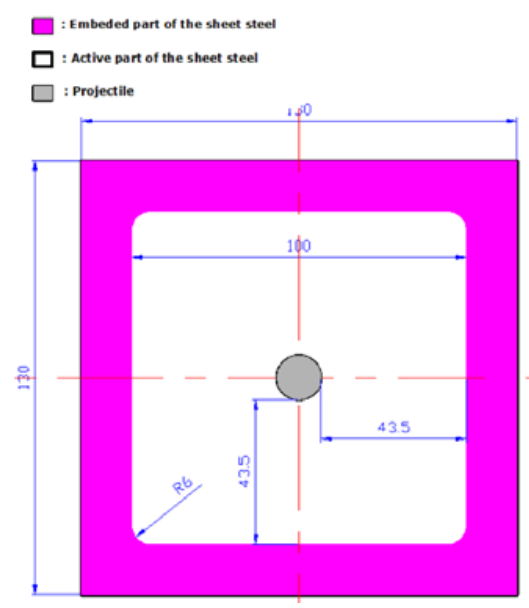

Fig. 1. Scheme of the samples used in the perforations tests.

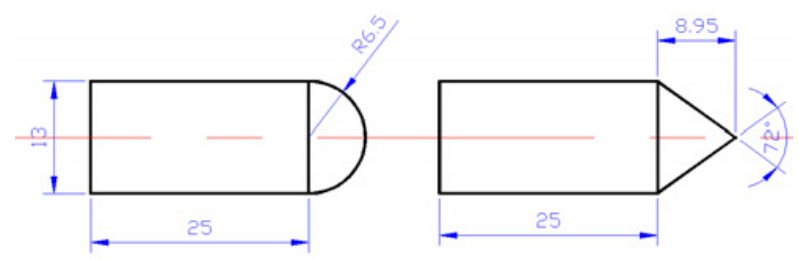

Fig. 2. Projectiles used in the perforations tests.

process. What is especially remarkable is the fact that, for this material, the phase transformation is observable during high rate loading events [3].

A scheme of the samples used in the perforation tests is shown in Fig. 1. The tested square sheets were $\mathrm{A}_{0}=130 \times 130 \mathrm{~mm}^{2}$ and their active surface after they were screwed and clamped on the support was $\mathrm{A}_{f}=$ $100 \times 100 \mathrm{~mm}^{2}$. Two different target thicknesses have been considered, $\mathrm{t}_{1}=0.5 \mathrm{~mm}$ and $\mathrm{t}_{2}=1.0 \mathrm{~mm}$. Hemispherical and conical projectiles have been used in the perforation tests. The mass of both projectile types was $\mathrm{M}_{\mathrm{p}}=30 \mathrm{~g}$; their geometry and dimensions are shown in Fig. 2. The projectiles were made of maraging steel which exhibits quite higher yield stress than that corresponding to the AISI 304 steel under dynamic conditions of deformation. In addition, the projectiles underwent a heat treatment in order to increase their hardness.

In order to perform perpendicular impact tests on the steel sheets a pneumatic gas gun has been used. Initial impact velocity $V_{0}$ and residual impact velocity $V_{r}$ were measured using lasers coupled to photodiodes and time counters. Next, the experimental results are discussed.

\section{Analysis of experimental results}

In Fig. 3 the experimental results obtained using the conical projectile configuration are depicted as residual velocity $V_{r}$ versus impact velocity $V_{0}$ curves. For the target thickness $\mathrm{t}_{1}=0.5 \mathrm{~mm}$ the ballistic limit found is $\mathrm{V}_{\mathrm{bl}} \approx 38 \mathrm{~m} / \mathrm{s}$. Due to the small thickness of the target, once the ballistic limit velocity is overpassed $\mathrm{V}_{0}>\mathrm{V}_{\mathrm{bl}}$, the curve $\mathrm{V}_{\mathrm{r}}-\mathrm{V}_{0}$ rapidly comes close to the condition determined by $V_{r}=V_{0}$. From this point on, inertia plays a

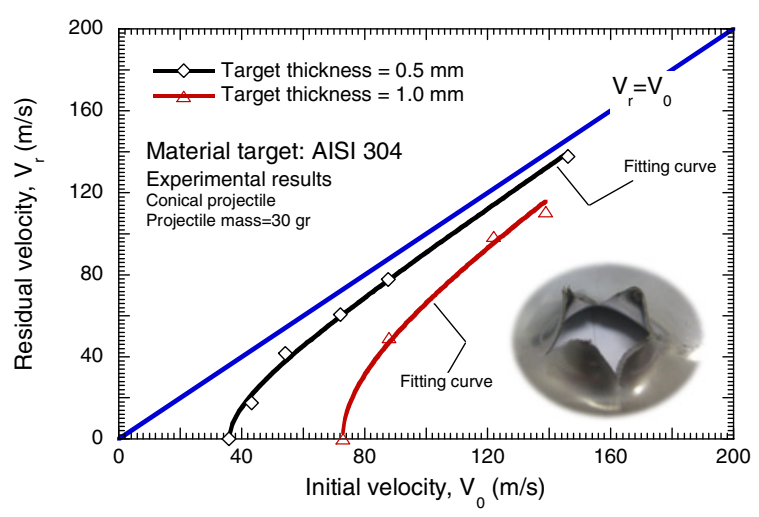

Fig. 3. Experimental results: conical projectile configuration. Residual velocity $V_{r}$ versus initial velocity $V_{0}$ for both target thicknesses analyzed, $\mathrm{t}_{1}=0.5 \mathrm{~mm}$ and $\mathrm{t}_{2}=1.0 \mathrm{~mm}$.

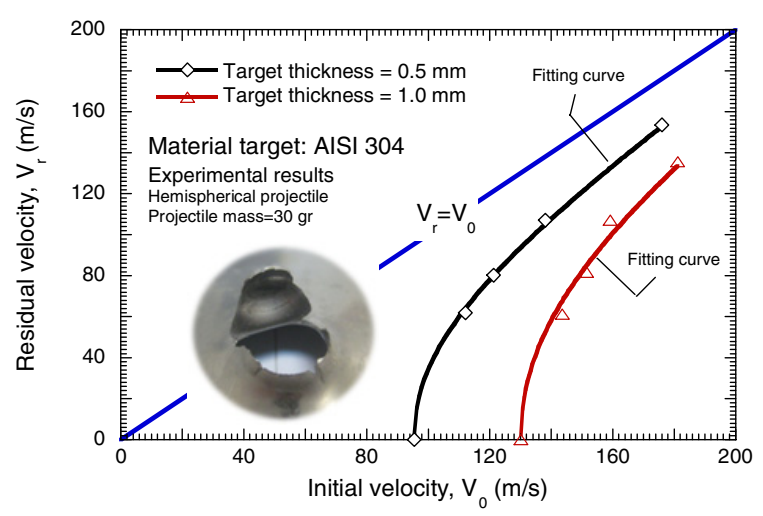

Fig. 4. Experimental results: hemispherical projectile configuration. Residual velocity $V_{\mathrm{r}}$ versus initial velocity $\mathrm{V}_{0}$ for both target thicknesses analyzed, $\mathrm{t}_{1}=0.5 \mathrm{~mm}$ and $\mathrm{t}_{2}=1.0 \mathrm{~mm}$.

key role in the process of energy absorption by the target. For the target thickness $\mathrm{t}_{2}=1.0 \mathrm{~mm}$ the ballistic limit registered is $\mathrm{V}_{\mathrm{bl}} \approx 75 \mathrm{~m} / \mathrm{s}$, which is almost double than that determined for the target thickness $t_{1}=0.5 \mathrm{~mm}$. Close to the ballistic limit condition, a rather small increase of the impact velocity leads to quite relevant rise of the residual velocity. In comparison to the target thickness configuration $\mathrm{t}_{2}=0.5 \mathrm{~mm}$, and within the range of impact velocities tested, the greater ability for energy absorption of the target in the case of $t_{2}=1.0 \mathrm{~mm}$ becomes manifest.

Moreover, it has been observed that petalling is the final stage of the perforation process for all the tests performed using the conical projectile configuration. The number of petals oscillates between three and five for all cases. Next, it has been examined if martensitic transformation occurs in the steel sheets during perforation. For that task the impacted plates have been cut; petals and cracking interfaces have been examined using a SEM. For the whole range of impact velocities covered, martensite has been found in the tested specimens.

In Fig. 4 the experimental results for the hemispherical projectile configuration are illustrated via $\mathrm{V}_{\mathrm{r}}-\mathrm{V}_{0}$ curves. For the target thickness $\mathrm{t}_{1}=0.5 \mathrm{~mm}$ the ballistic limit is $\mathrm{V}_{\mathrm{bl}} \approx 95 \mathrm{~m} / \mathrm{s}$ and for $\mathrm{t}_{2}=1.0 \mathrm{~mm}$ is $\mathrm{V}_{\mathrm{bl}} \approx 130 \mathrm{~m} / \mathrm{s}$.

In comparison with the conical projectile configuration the ballistic limit has considerably increased; the 


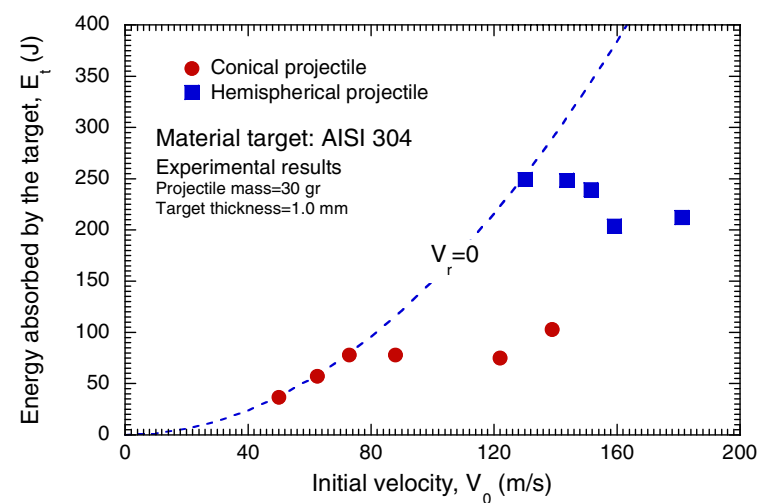

Fig. 5. Absorbed energy $E_{t}$ versus initial velocity $V_{0}$, for conical and hemispherical projectiles (1 $\mathrm{mm}$ thickness).

hemispherical projectile is revealed less efficient than the conical one to perforate the steel targets. Within the range of impact velocities tested and, for both target thicknesses examined, the $\mathrm{V}_{\mathrm{r}}-\mathrm{V}_{0}$ curves remain far from the condition determined by $\mathrm{V}_{\mathrm{r}}=\mathrm{V}_{0}$. The ability of the target to store energy in form of plastic deformation is larger when the hemispherical impactors are used. For the hemispherical projectile configuration, the large bending of the steel sheets during perforation leads to considerable radial sliding projectile/plate. Then, an extended targetzone subjected to severe plastic deformation is observed. Necking formation and subsequent propagation of radial cracks is the recurrent failure mode of the plates. The target-zone surrounding the necking is examined using a SEM. For the whole range of impact velocities covered, martensite has been found in the tested specimens. It was concluded that the amount of martensite observed is larger than that registered in the plates impacted by conical projectiles. In addition, the extension of the target in which martensite is detected is larger than that determined for the conical projectile configuration. This fact becomes evident if one bears in mind that the target-zone subjected to severe plastic deformation is larger if hemispherical impactors are used.

Fig. 5 shows, for both projectiles, the energy absorbed by the target versus the impact velocity for the $1 \mathrm{~mm}$ thickness sheet. For the hemispherical shape, the energy is much higher compared to that absorbed by the plate in case of the other projectile. Besides the greater difficulty of the hemispherical shape to perforate the sheet, hardening due to phase transformation plays in this case an important role in the energy absorption. Contrarily, martensite hardly develops in a sheet impacted by the conical projectile due to its ability to rapidly perforate the target.

\section{Constitutive model}

The constitutive description is based on the previous works of Olson and Cohen [4], Stringfellow et al. [5] and Papatriantafillou et al. [6], developed to account for SIMT in steels containing metastable austenite, and adds the following features:

- Instead of the polynomial expression applied by other authors to describe the change with temperature $\theta$ of the parameter $\alpha$ (proposed by Olson \& Cohen [4] to calculate the increase of shear bands in austenite due to plastic slip), it considers an exponential law. This law better captures the decrease of the transformation rate with increasing temperature within the temperature range in which the SIMT occurs.

- The linearly temperature dependent formulation proposed by Stringfellow et al. [6] for the thermodynamic driving force (used to describe martensite formation under static loading) is replaced by an exponential equation. The new law provides greater temperature sensitivity of the rate of martensitic transformation. This becomes relevant at high strain rates where the temperature rise of the material results from the adiabatic character of the plastic deformation. According to the experimental results reported by RodríguezMartínez et al. [7] at high deformation rates (and therefore under significant temperature increase), small variations on the rise of temperature lead to relevant differences on the volume fraction of martensite formed.

- Plastic deformation in both austenite and martensite are driven by a plastic potential including thermal effects. This potential leads to a power yield law for both viscous and thermal effects, commonly accepted to model strain rate hardening and temperature softening in metals and alloys. The effective properties of viscoplastic heterogeneous materials are calculated with the modified secant method proposed by Suquet [8] which has been extended for thermal softening effects.

- The temperature increase under adiabatic conditions has been calculated through the first principle of thermodynamics. Since the most widely used value of the Quinney-Taylor coefficient for steels is $\eta=0.9$ and the upper value of latent heat in dynamic tensile test is close to $10 \%$ compared to the average temperature rise due to plastic work (according to the estimation by Rusinek and Klepaczko [9]) a value of $\eta=1$ has been chosen to consider both sources of heat: the conversion of plastic work and the martensitic transformation.

Finally, the model links a law for the kinetics of SIMT, a thermoviscoplastic law for each phase (austenite and martensite) and a homogenization method to obtain equivalent properties. The equations are discretized using an explicit algorithm and the model is implemented in the user subroutine of ABAQUS/Explicit finite element code. A full description of the model and the corresponding integration algorithm can be found in [8].

\section{Finite element simulation of the perforation process of AISI 304 steel sheets}

A Lagrangian 3D finite element model for simulation of the perforation process of the AISI 304 steel sheets has been built in ABAQUS/Explicit. A fully 3D configuration allows describing radial cracking and petalling failure mode which characterizes the perforation of metallic sheets by conical and hemispherical projectiles. 


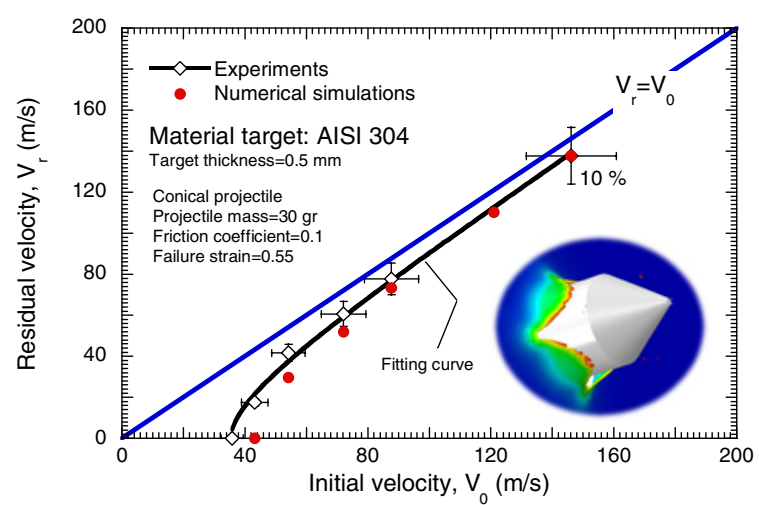

(a)

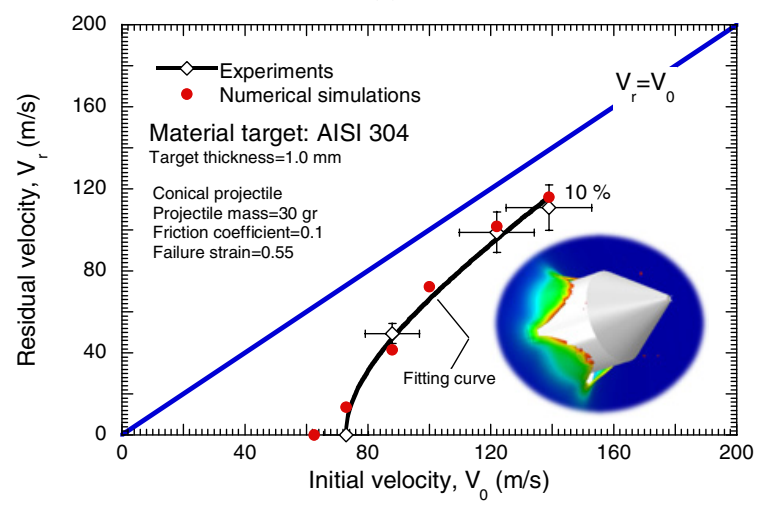

(b)

Fig. 6. Conical projectile configuration. Residual velocity $V_{r}$ versus initial velocity $\mathrm{V}_{0}$. Comparison between experiments and numerical simulations. (a) Target thickness $t_{1}=0.5 \mathrm{~mm}$, (b) Target thickness $\mathrm{t}_{2}=1.0 \mathrm{~mm}$.

According to the experimental evidence which revealed absence of erosion on the projectile-surface after the impact, the projectile has been defined as a rigid body. It enables to reduce the computational time required for the simulations. A constant value of the Coulomb friction coefficient $\mu=0.1$, corresponding to a dry steelstainless steel contact, has been used to define the contact projectile/plate.

In order to simulate the perforation process within a Lagrangian framework a failure criterion must be considered. The use of failure criteria founded on the material deformation behaviour is a practice widely extended in the analysis of metallic structures subjected to dynamic loads. In this work the material failure is defined by a constant value of the equivalent plastic strain, assuming a tensile stress state during failure. The value of the failure strain adopted in the simulations, $\varepsilon_{\mathrm{f}}=0.55$, was defined through an optimization process oriented to the lower error level. This value is slightly higher than that corresponding to the necking strain under dynamic loading [7]. This adequately helps to define in the FE simulations the process of strain localization and necking formation which leads to the target collapse. Next, the comparison between experiments and finite element simulations is conducted.

So, let us start analyzing the FE results for the conical projectile configuration. Fig. 6 shows the comparison between experiments and numerical simulations in terms of

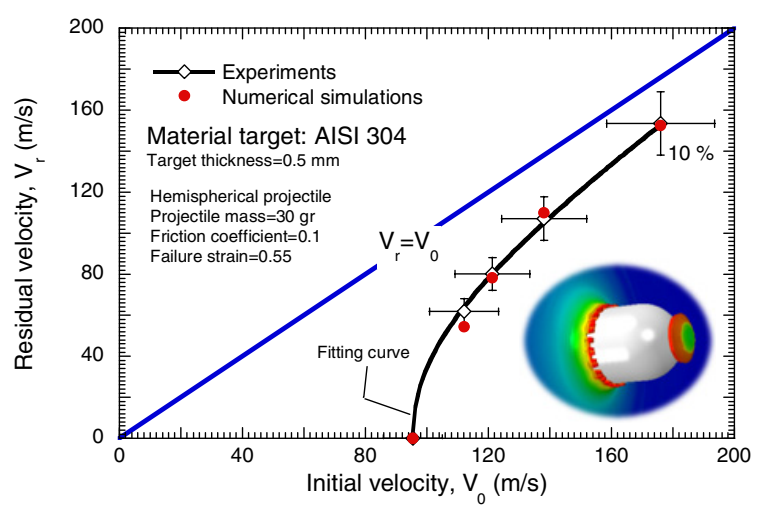

(a)

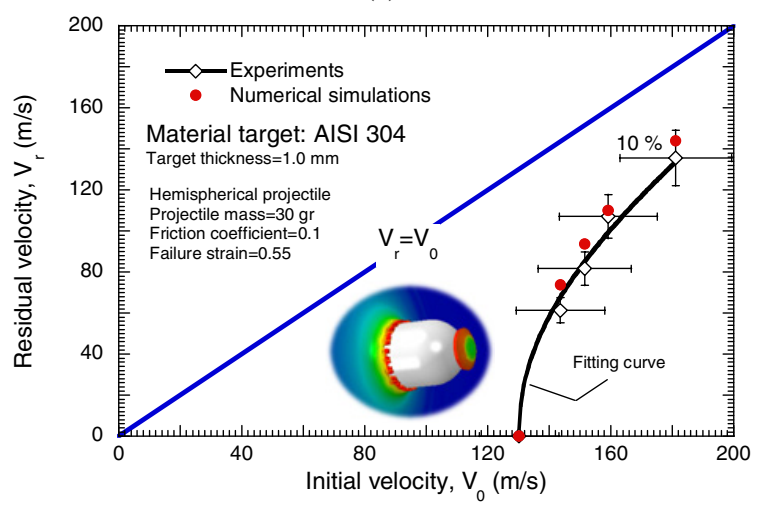

(b)

Fig. 7. Hemispherical projectile configuration. Residual velocity $V_{r}$ versus initial velocity $V_{0}$. Comparison between experiments and numerical simulations. (a) Target thickness $t_{1}=0.5 \mathrm{~mm}$, (b) Target thickness $\mathrm{t}_{2}=1.0 \mathrm{~mm}$.

$\mathrm{V}_{\mathrm{r}}-\mathrm{V}_{0}$ curves for both target thicknesses examined. It can be observed that the agreement is rather satisfactory. The difference being less than $15 \%$ within the whole range of impact velocities considered.

Moreover, the model is able to predict the characteristic failure mode of the plates. Petalling formation occurs at the final stage of the perforation process within the whole range of impact velocities examined. Next, let us focus our attention on the $\gamma \rightarrow \alpha^{\prime}$ transformation predicted by the FE analyses. The model predicts the formation of martensite within a narrow zone surrounding the impact location. In particular, the SIMT process is primed in cracking interfaces and petals. Then, two interesting findings have to be remarked:

- The amount of martensite decreases with impact velocity due to the process of adiabaticity.

- The amount of martensite increases with target thickness

These observations are in close agreement with the experimental evidence.

Next, the FE results obtained for the hemispherical projectile configuration are presented. Fig. 7 shows the comparison between experiments and numerical simulations in terms of $\mathrm{V}_{\mathrm{r}}-\mathrm{V}_{0}$ curves for both target thicknesses examined. As discussed for the conical projectile 
configuration the agreement is rather satisfactory, the difference being less than $10 \%$ within the whole range of impact velocities considered. The ballistic limit value is adequately captured.

Furthermore, the model also succeeds in defining the failure mechanisms associated to this projectile-target configuration. Necking and radial cracking around perforations is observed for the whole range of impact velocities and both target thicknesses investigated. It should be noticed that the computational model predicts the formation of martensite during perforation within the whole range of impact velocities examined for both target thicknesses considered. The SIMT process is primed in cracking interfaces and petals (as reported for the conical projectile configuration), but relevant quantities of martensite can be also detected within a wide area around the impact location. The amount of martensite predicted in the FE analyses is greater for the hemispherical projectile configuration than for the conical one. This observation matches with the experimental evidence described in section 3 .

\section{Conclusions}

In this work the attention has been focused on examining the mechanical behaviour of the AISI 304 metastable austenitic steel subjected to perforation. This steel is considered to be the reference metastable austenitic stainless steel for studying the SIMT process. Moreover, it has the ability to transform to martensite even at high strain rates.

Perforation tests have been conducted using a pneumatic cannon within the range of impact velocities $35 \mathrm{~m} / \mathrm{s}$ $<\mathrm{V}_{0}<200 \mathrm{~m} / \mathrm{s}$. Two projectile nose-shapes, conical and hemispherical, and two target thicknesses, $\mathrm{t}_{1}=0.5 \mathrm{~mm}$ and $t_{2}=1.0 \mathrm{~mm}$, have been considered. The effect of the projectile nose shape on the ability of the target for energy absorption has been evaluated. The tested samples have been examined using a SEM and martensite has been detected in all the specimens impacted. The role played by the projectile nose shape on the transformation is highlighted.

As a means of delving further into the SIMT process which occurs during the perforations, a three dimensional model is built in ABAQUS/Explicit to simulate the experiments. The thermo-viscoplastic material response is defined by the constitutive model developed by the authors elsewhere [10]. The numerical results are compared with the experiments and satisfactory matching is obtained for the projectile-target configurations examined. The analysis allows deriving a rational explanation for the $\gamma \rightarrow \alpha$, transformation observed in AISI 304 steel sheets subjected to high rate loading processes. The threshold material straining required to overcome the activation barrier between austenite and martensite is lower than the material straining required to reach the limiting level of material temperature which inhibits the transformation. Moreover, the numerical model succeeds in describing the perforation mechanisms associated to each projectile-target configuration analyzed.

\section{Acknowledgements}

A. Rusinek thanks M. Tavian technician in LaBPS-ENIM for his contribution concerning the development of the velocity sensors.

The researchers of the University Carlos III of Madrid are indebted to the Ministerio de Ciencia e Innovación de España (Project DPI2011-24068) for the financial support received which allowed conducting part of this work.

\section{References}

1. G. Corbett, S. Reid, W. Johnson, Int J Impact Engng 18, (1996)

2. J. Dean, C. Dunleavy, P. Brown, T. Clyne, Int J Impact Engng 36, (2009)

3. J.A. Rodríguez-Martínez, A. Rusinek, R. Pesci, ThinWalled Struct 48, (2010)

4. G.B. Olson, M. Cohen, Metal Trans A 6A, (1975)

5. R.G. Stringfellow, D.M. Parks, G.B. Olson, G. B., Acta Metall Mater 40, (1992)

6. I. Papatriantafillou, N. Aravas, G. Haidemenopoulos, Steel Research Int. 75, (2004)

7. J.A. Rodríguez-Martínez, R. Pesci, A., Rusinek, Mat Sci Engng A 528, (2011)

8. P. Suquet, (IUTAM Symposium, Kluwer, 1996)

9. A. Rusinek, J.R. Klepaczko, Materials and Design 30, (2009)

10. R. Zaera, J.A. Rodríguez-Martínez, A. Casado, J. Fernández-Sáez, A. Rusinek, R. Pesci, R., Int J Plast 29, (2012) 